\title{
Low Frequency Electrical Resonance in Water
}

\begin{abstract}
We report the observation of sharp electrical resonance of water with width $\sim 2 \mathrm{neV}$ in the low radio frequency range at room temperature. The neV level of the resonant width under room temperature $(\sim 25$ $\mathrm{meV}$ ) is consistent with the theory in Wang et al (2020) that predicts a macroscopic long-range coherent quantum mechanical excited states, Majorana fermions, resulting from quantum entanglement of proton hopping at hydrogen bonds.
\end{abstract}

\section{INTRODUCTION}

Wang et al (2020) propose a general variational fermionic many-body wavefunction that generates an effective Hamiltonian in quadratic form which can then be exactly solved. The theory is applied to water molecules and predicts a sharp electrical resonant absorption at the frequency $\Delta / \hbar$, where $\Delta$ is expected to be very sensitive to local $\mathrm{pH}$ levels in a heterogeneous aqueous system, as well as the details in the hydrogen bond and the strength of spin-orbit coupling of protons at the hydrogen bond.

In this paper, we carried out the experiments to confirm the predictions from the theory. We report the observation of sharp electrical resonance of water in the low radio frequency range at room temperature. The sharpest resonance has a half width less than $0.5 \mathrm{MHz}$, i.e., $2 \mathrm{neV}$, suggesting that the lifetime of the resonant excitation to be in the order of milliseconds or longer.

We will first present the experimental setup, then show the sharp resonance observed for distilled water. The sensitivity of the resonance on bias voltage, $\mathrm{pH}$ level (proton doping), temperature, structural ordering, and other boundary conditions are discussed. We also show that L-Tryptophan (an amino acid) water solution greatly enhanced resonance amplitude and show much narrower width than the distilled water.

Dielectric properties of water and aqueous solutions have been the subject of many theoretical and experimental studies. The starting points of our study is to conduct an extensively literature review, focusing on the prior experimental studies measuring the complex permittivity of water or aqueous solutions, in particular the frequencies at which the experiments were conducted. Haggis et al (1952) measured dielectric properties of water and aqueous solutions of ions and molecules. Their experiments were conducted at three frequencies between $3-30 \mathrm{GHz}$ (wavelength $9.22 \mathrm{~cm}, 3.175 \mathrm{~cm}$, and $1.264 \mathrm{~cm}$ ). Malmberg and Maryott (1956) measures dielectric constant of distilled water from $0^{\circ} \mathrm{C}$ to $100^{\circ} \mathrm{C}$, at frequency of 3,6,12,24, 48, and $96 \mathrm{KHz}$. Schwan et al (1976) measures permittivity of water at frequencies from $100 \mathrm{MHz}$ to $3 \mathrm{GHz}$. Kaatze (1989) measures complex permittivity values for pure water between $0^{\circ} \mathrm{C}$ and $50^{\circ} \mathrm{C}$ and at frequencies from 1.1 to $57 \mathrm{GHz}$. Rusiniak (2004) studies the electric properties of distilled water from $5 \mathrm{~Hz}$ to $13 \mathrm{MHz}$ frequency range between $20-90^{\circ} \mathrm{C}$ temperature range. Gregory and Clarke (2005) measures static permittivity of eight polar liquids and two non-polar liquids at temperatures from 5 to $50^{\circ} \mathrm{C}$ and at a number of frequencies below $1 \mathrm{MHz}$. Shi et al (2014) studies the dielectric constant of water and Ice Ih at low infrared spectrum, focusing on explaining the peaks observed at $\sim 200 \mathrm{~cm}^{-1}$ and $\sim 700 \mathrm{~cm}^{-1}$. Silva et al. (2017) reports the electrical and dielectric properties of water in liquid phase, such as impedance, electric conductive, dielectric permittivity, molar polarization and dipole moment as a function of low frequency $(1-20 \mathrm{KHz})$ in the 288-314 $\mathrm{K}$ temperature range. Zhuravlev et al (2018) studies electrophysical properties, including complex permittivity of distilled water as well as water 
with different conductive impurities. Their measurements were taken at both low frequency $(20 \mathrm{~Hz}$ to $2 \mathrm{MHz}$ ) and high frequency $(500 \mathrm{MHz}$ to $40 \mathrm{GHz})$.

To summarize, our literature review did not find any prior experimental work that has discovered a low frequency resonance as predicted in Wage (et al). In addition, we found that the frequency band between $13 \mathrm{MHz}$ and $100 \mathrm{MHz}$ has not been experimented previously and thus this frequency band is the focus of our current work.

\section{METHODS}

The apparatus, shown in Figure 1, can be generally described as composing of three components: electrical signal generator(s), a test device in water or aqueous solution, and measuring instrument(s) analyzing the response signals. For our experiment, we use two waveform function generators (Siglent SDG2122x and Siglent SDG2042x) to generate the electrical signals and an oscilloscope (Siglent SDS1104xe) for response measurement. We built the test devices as a pair of parallel gold-plated metal plates bound closely with plastic foil (GLAD Cling Wrap), separated by a thin layer of the same plastic on both ends of the plates to allow air or water filled in-between the plates. In this setup, the parallel metal plate and the water in-between function as a capacitor in the circuit.

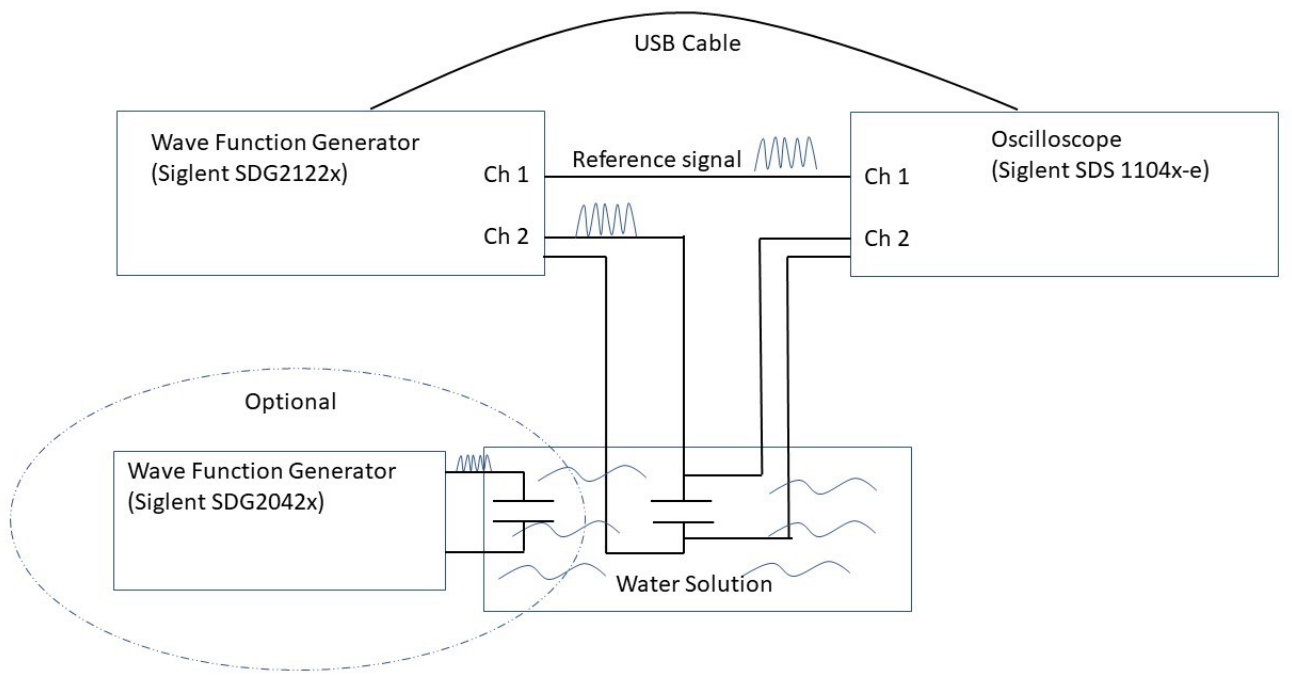

Figure 1. Experiment setup for our experiment

Note that in Figure 1, the waveform generator and the oscilloscope are connected by a USB cable, and a reference signal is sent from Channel 1 of the generator to Channel 1 of the oscilloscope. This connection is to enable the built-in Bode Plot function of the Oscilloscope. Siglent website provides a description of the connection as well as an example of using the Bode Plot function for testing a low pass filter ${ }^{1}$.

Built-in Bode Plot function of the oscilloscope (Siglent 1104x-e) was used to analyze the amplitude and phase changes caused by the capacitive effects of the parallel plates when the plates were subject to different electrical frequencies as they were submerged in various environments: open air, distilled water, or different water solutions. The amplitude in the Bode Plot (in $\mathrm{db}$ ) measures the strength of the signal through the test device connected in the Channel 2 of the waveform generator relative to the strength of

\footnotetext{
${ }^{1}$ https://siglentna.com/application-note/bode-plot-filter-oscilloscope-generator/
} 
the reference signal in Channel 1. Higher amplitude indicates stronger current and thus lower impedance of the test device. The measurement data were collected with frequencies ranging from $10 \mathrm{~Hz}$ to $100 \mathrm{MHz}$, the upper limit of the Siglent SDG2122x frequency range.

\section{RESULTS}

\section{Resonance in Distilled Water}

To establish a baseline profile, we measured the test subject's amplitude and phase responses to electrical frequencies in open air ("Open Air", dashed lines in Figure 2A). The test subject was then submerged in distilled water. Frequency responses were again recorded (solid lines in Figure 2A). Compared to the "Open Air" baseline, we observed a sharp ramp-up of amplitude, peaking around 34.27 MHz. An abrupt change in phase was also observed neighboring that frequency.

In addition to the sharp resonance observed at $34.27 \mathrm{MHz}$, we also noted some small spikes at higher frequencies around $50 \mathrm{MHz}, 72 \mathrm{MHz}$, and $92 \mathrm{MHz}$, with 50 and $72 \mathrm{MHz}$ also observed in air sample.
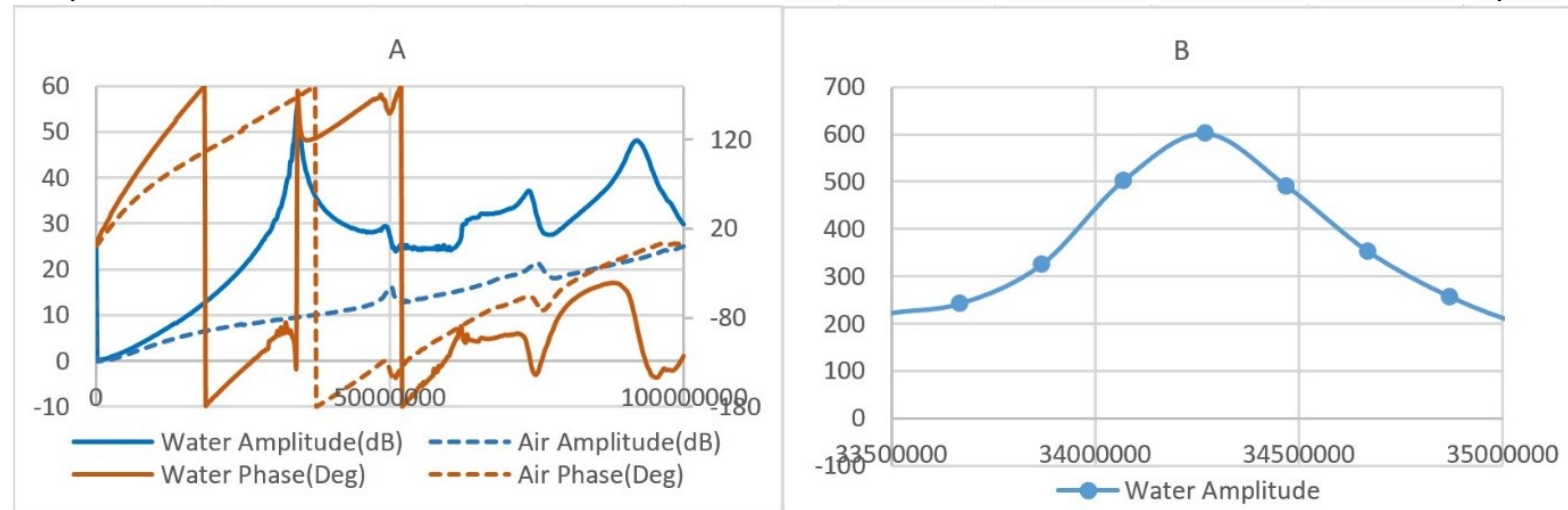

Figure 2. A. Left panel is the full spectrum, air data is plotted as dashed lines for comparison. B. Right panel is the zoomed in view of the peak at $34.27 \mathrm{MHz}$. The lifetime of the resonance is estimated to be $\sim 1 /(0.4$ $\mathrm{MHz}$ ), i.e. 2.5 millisecond.

The sharp changes in the amplitude and the phase point to a change in the permittivity of water caused by a resonant response of water around the resonant frequency. Resonant response of water in this low frequency range has not been documented before, and is consistent with the prediction in Wang et al (2020)..

\section{Sensitivities of the Resonance on Various Environment/Sample Factors}

We have noticed that the sharp resonant feature observed can be very sensitive to the sample geometric characteristics, for example, the distance between the two plates. In addition, the resonant feature observed is also sensitive to various parameters: sample material, temperature of the water, $\mathrm{pH}$ level of the water, bias DC voltage across the metallic plates, and most significantly, amino acid solvent in water seems to greatly enhance the resonant signal toward much longer resonant lifetime and higher dielectric response amplitude.

\section{Sensitivities on Inter-Plate Distance}

We have noticed that for the two gold plate samples (rectangular $\sim 5 \mathrm{~mm} \times 25 \mathrm{~mm}$ ), the sharpest resonant frequency shifts lower with lower inter-plate distance. We confirmed this observation (see Figure 3 ) 
qualitatively by using two larger ( $20 \mathrm{~mm}$ diameter) circular plates and adjust the inter-plate distance hanging from a plastic string without any GLAD Wrap bound.

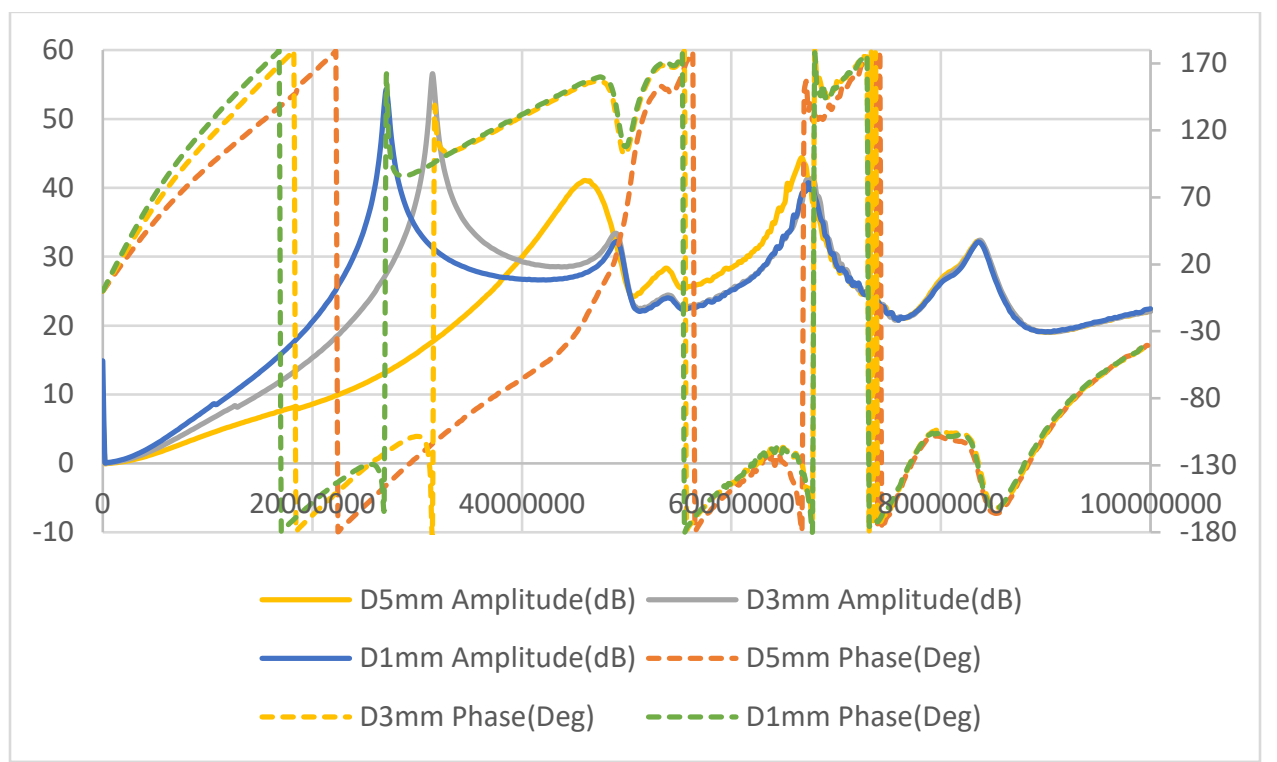

Figure 3. Resonant frequency becomes lower as inter-plate distance shortens.

We also noted that the higher frequency features are more stable than the sharpest resonance. It is important to emphasize that this frequency dependence on inter-plate distance cannot be attributed to higher field strength of the applied (zero DC bias) electric field, as we have confirmed that for a wide range of applied voltage $(0.004 \mathrm{~V}$ to $4 \mathrm{~V})$, the in-situ resonant frequency does not change.

\section{Sensitivities on $\mathrm{pH}$ and Temperature}

We note that the distilled water under temperature around 20 degree Celsius has a $\mathrm{pH}$ value $\sim 5.6$ due to the carbon dioxide solvent in water. To change the $\mathrm{pH}$ for pure water, we boiled the distilled water and take measurements of the Bode Plot for each sweep. At the start of the measurement, the temperature of the water is 79 degree Celsius with $\mathrm{pH}$ at 6.9. At the end of each sweep/scan of the Bode Plot, we take down the two sharpest resonant frequencies and corresponding amplitudes, in addition to the $\mathrm{pH}$ value and the temperature of the water. Figure 4 shows the sharpest resonant frequency vs temperature and $\mathrm{pH}$.

A

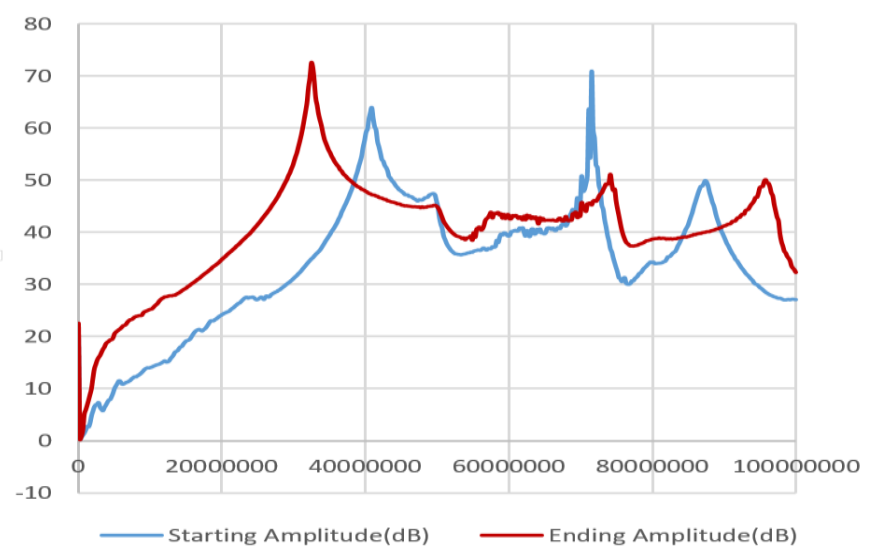

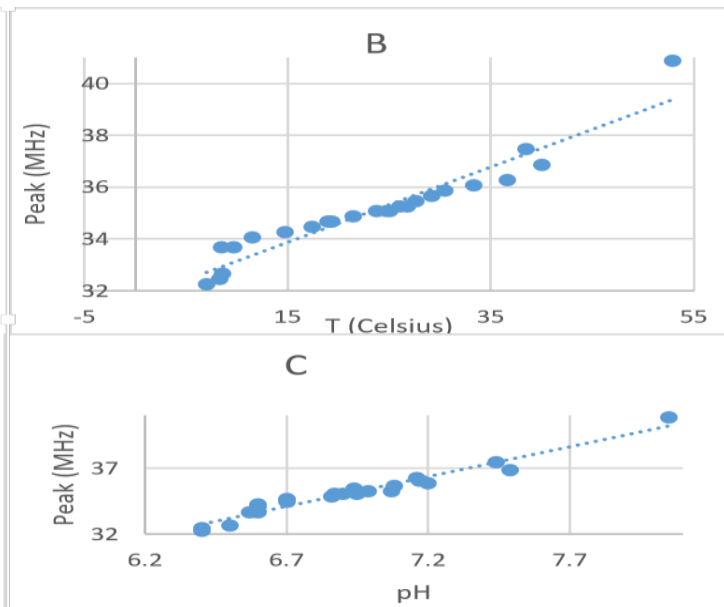


Figure 4. A: Starting with a sharp high frequency peak at high temperature, ends with a sharp peak at lower frequency. B: Lower Peak position vs Temperature. C: Lower peak position vs pH

Note that it is hard to separate the impact of temperature and $\mathrm{pH}$ from these results as there is a strong collinearity between the $\mathrm{pH}$ and temperature.

To probe the temperature dependence below or around the freezing temperature, we freeze the same sample in freezer set at -32 Celsius. We start the Bode Plot after the sample is taken out of the freezer. We measured $\mathrm{pH}$ and water temperature after the last sweep of data taken and their respective values are 6.5 for $\mathrm{pH}$ and 8.1 Celsius for temperature. We observed (see Figure 5) that no resonance is observed at first sweep, and it starts to appear at higher frequency. As the sample starts to warm up, the peak starts to sharpen and peak frequency drifts from $\sim 43 \mathrm{MHz}$ to $32.26 \mathrm{MHz}$ at the last sweep. This suggests a different temperature dependence at lower temperature than at higher temperature. If one recalls that inter-molecular distance of water is the smallest at around 4 degree Celsius, the change of temperature dependence for the resonant frequency seems to coincide with the change of thermal expansion coefficient of water. We also want to point out that around freezing temperature, a first order structural phase transition also kicks in and our current experimental results cannot conclusively separate all these factors (temperature, $\mathrm{pH}$, and structural ordering) on the resonant feature observed. Resonant feature start to stabilize after Sweep 10 where we start to see ice melting at the contact with the metallic plates.

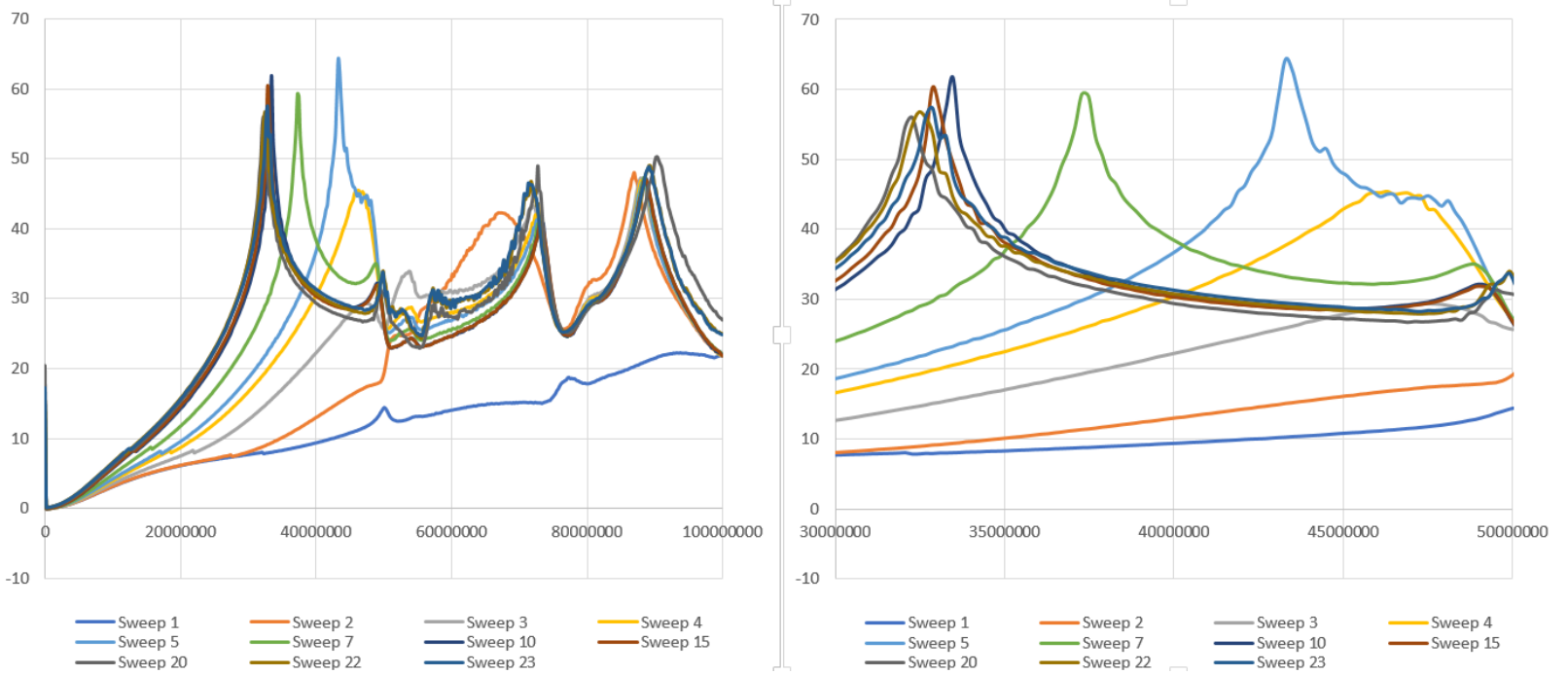

Figure 5. The lowest lying curve is the first sweep which corresponds to lowest ice temperature where no resonance features are observed. Sweep 2 (gray line) some high frequency resonances start to form. The low frequency feature starts to sharpen at sweep 5, and gradually moved lower to 32.26 but comes back up to 32.87 at the last two sweeps (right panel for a zoomed-in view).

\section{Sensitivities on Bias DC Voltage}

When we change the bias during this experiment, we notice that it takes a long time for the Bode Plot data to stabilize, sometimes as long as over two hours. This is not surprising consider that DC bias mainly tries to align the micro domains of water molecules responsible for the resonance. Figure $6 \mathrm{~A}$ shows the stabilized resonant frequency versus bias DC voltage and Figure $6 \mathrm{~B}$ shows at Bias Voltage at $1.5 \mathrm{~V}$, the resonant frequency oscillates over time. 
A

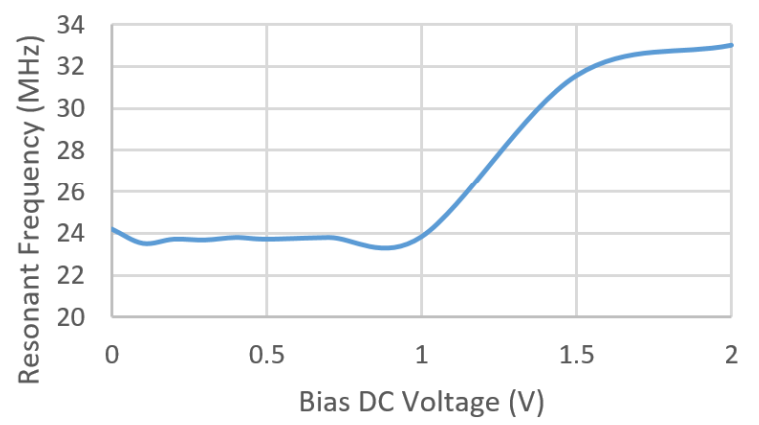

B

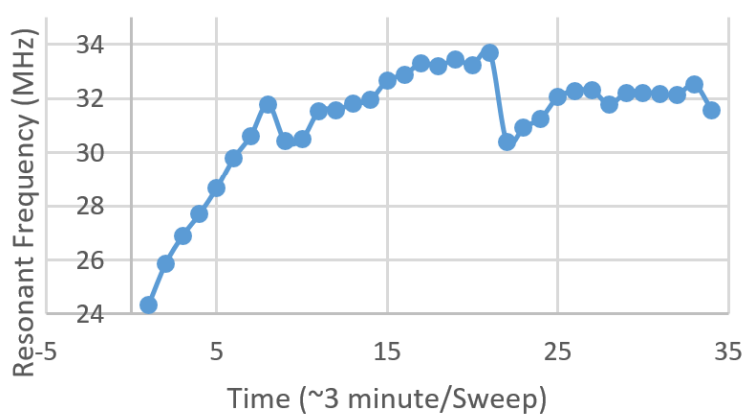

Figure 6. A: resonant frequency versus bias DC voltage. B: at bias DC voltage $1.5 \mathrm{~V}$, the resonant frequency oscillates over time and slowly stabilized.

\section{Observation of Resonant Response from Second Signal Generator}

As shown in Figure 1, we put a second metallic plates in the water that is connected only to the second signal generator. The signal collected for sample 1 will reflect the cross talk effect due to the second signal generator. The second signal generator is at a fixed frequency of $32.26 \mathrm{MHz}$, which is the resonant frequency of the sample 1 before we put the sample 2 in the water. The sample 2 voltage is set at $4 \mathrm{~V}$, and we change the sample 1 voltages at $4 \mathrm{mV}, 40 \mathrm{mV}$, and $400 \mathrm{mV}$. Figure 7 shows the three different sample 1 voltage results with $7 \mathrm{~A}$ the full spectrum, and 7B the zoomed-in view around $32.26 \mathrm{MHz}$. The impact of crosstalk from the second signal is evident and it depends on the relative amplitude between the two signals, as expected. Note that except for more noises in the data, the resonant frequency of the original sample 1 does not change with the voltage applied on itself. We also note that the relative orientation of the two sets of samples do not seem to matter that much.

A

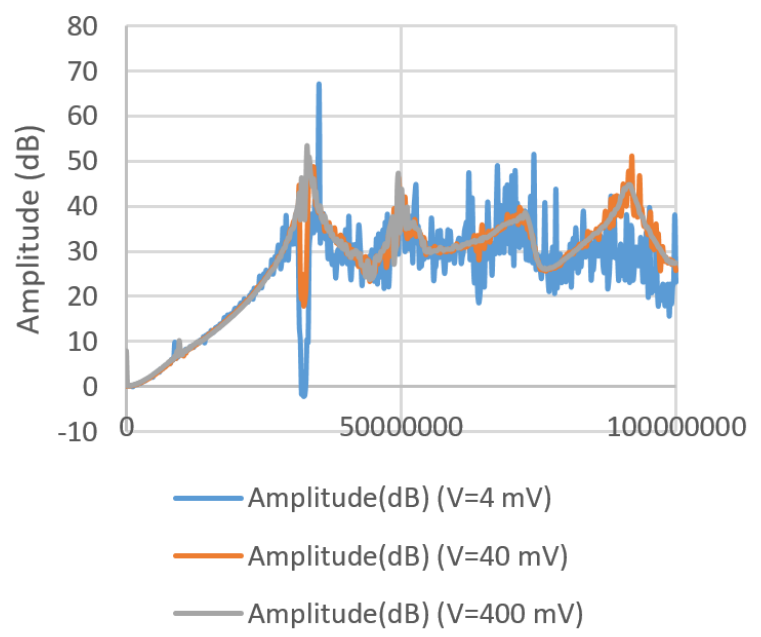

B

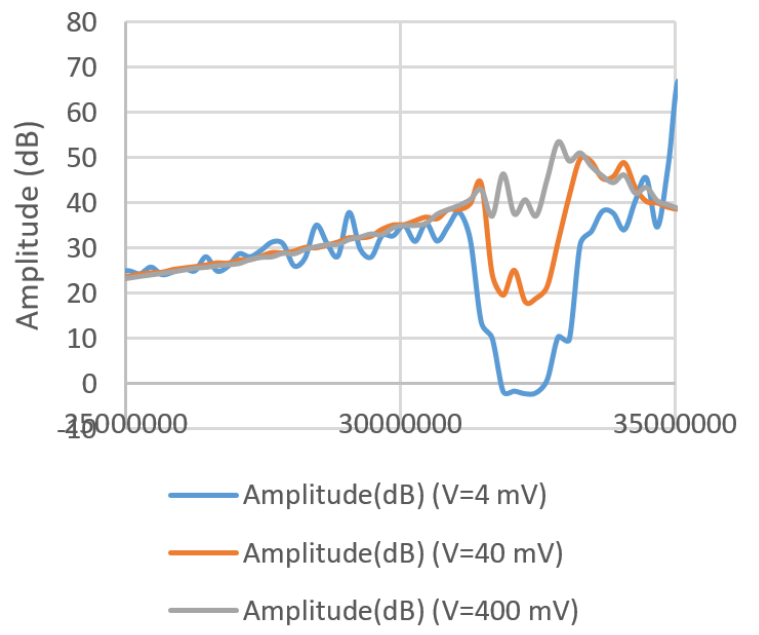

Figure 7. Three different sample 1 voltage $(4 \mathrm{mV}, 40 \mathrm{mV}, 400 \mathrm{mV})$ results with $7 \mathrm{~A}$ the full spectrum, and $7 \mathrm{~B}$ the zoomed-in view around $32.26 \mathrm{MHz}$, the fixed frequency of the second generator.

\section{Observation of Resonant Response on Amino Acid Water Solution}

Finally, we want to show that some amino acid molecules have dramatic impact on the amplitude at the resonant frequency, even though the $\mathrm{pH}$ value does not seem to differ very much. We stir the water 
solution fully until the amino-acid molecules reaches its maximum solubility. Figure 8 shows the comparison between distilled water and fully saturated L-Tryptophan (purity $>99 \%$ ) water solution. We plot the amplitude in $\left(10^{\wedge}(\mathrm{dB} / 20)\right)$ to draw attention to the dramatic increase of the resonance magnitude.

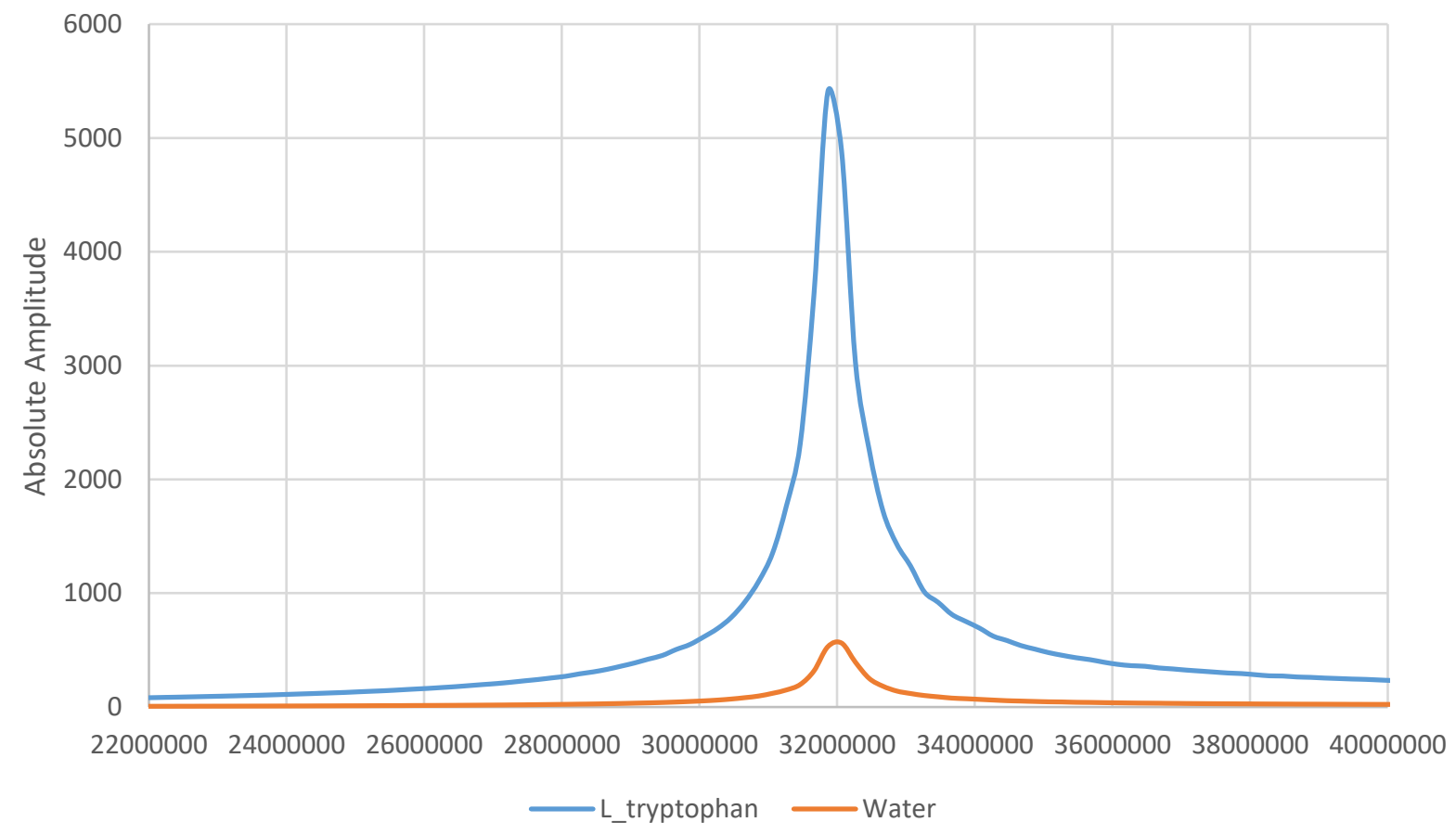

Figure 8. Comparison of resonance magnitude between distilled water (orange line) and fully saturated LTryptophan amino-acid solution.

\section{DISCUSSION}

We report the observation of sharp electrical resonance(s) of water in the low radio frequency range at room temperature. The sharpest resonance has a half width less than $0.5 \mathrm{MHz}$, i.e., 2 neV, suggesting that the lifetime of the resonant excitation to be in the order of higher than milliseconds. This long lifetime of the observed resonance has not been documented before and is consistent with the theory in Wang et al (2020) that predicts a macroscopic long-range coherent quantum mechanical excited state resulting from proton hoping at hydrogen bonds..

DATA AVAILABILITY

Data available upon reasonable request.

\section{REFERENCES}

Gregory, A., \& Clarke, R. (2005). Traceable measurements of the static permittivity of dielectric reference liquids over the temperature range 5-50 ${ }^{\circ} \mathrm{C}$. Meas. Sci. Technol., (16) 1506-1516.

Haggis, G., Hasted, J., \& Buchanan, T. (1952). The dielectric properties of water in solutions. J. Chem. Phys., (20)9 1452.

Kaatze, U. (1989). Complex permittivity of water as a function of frequency and temperature. J. Chem. Eng. Data, (34)4 371-374.

Malmberg, C., \& Maryott, A. (1956). Dielectric constant of water from 0 to $100 \mathrm{oC}$. J. Research of National Bureau of Standards, (56) Research paper 2641. 
Rusiniak, L. (2004). Electric properties of water. New experimental data in the $5 \mathrm{~Hz}-13 \mathrm{MHz}$ frequency range. Acta Geophys, (52) 12004.

Schwan, H., Sheppard, R., \& Grant, E. (1976). Complex permittivity of water at $25^{\circ} \mathrm{C}$. J. Chem. Phys., (64) 2257-2258.

Shi, L., Ni, Y., Drews, S., \& Skinner, J. (2014). Dielectric constant and low-frequency infrared spectra for liquid water and ice Ih within the E3B Model. J. Chem. Phys., 141(8).

Silva, L. d., Freitas, K., \& Sousa, F. d. (2017). Electrical and dielectric properties of water. Scientia Plena, (13) 1.

Wang, X., Chen, X., Ke, L., Cheng, H.-P., \& Harmon, B. N. (2020). Exact Self-Consistent Effective Hamiltonian Theory. http://arxiv.org/abs/2010.15192.

Zhuravlev, V., Suslyaev, V., Zhuravlev, A., \& Korovin, E. (2018). Analysis of dielectric spectra of water with conductive impurities in a wide frequency range. Russian Phyics J., (60)11, 1893-1900. 